\title{
Dossier Évolution et créationnisme La théorie de l'évolution face au créationnisme
}

\author{
Anne-Françoise Schmid
}

Le créationnisme, mouvement qui a depuis longtemps une importance aux États-Unis, arrive actuellement en Europe par le Nord. Ainsi, il devient courant de rencontrer sur les campus des étudiants, même biologistes, qui sont créationnistes. Certains d'entre eux opposent la Bible à Darwin, nient l'évolution au profit de la création, d'autres affirment que le monde est trop complexe pour ne pas avoir été créé par un être intelligent. Cette seconde position est une forme adoucie du créationnisme d'origine, connue sous le terme d'Intelligent Design (ID). Une question critique se pose donc : pourquoi cette offensive anti-évolution de la part des intégrismes des religions "abrahamiques" ? Il est possible qu'avec les crises politiques et économiques, les religions du livre deviennent plus indépendantes des contextes sociaux et locaux, et donc plus " universelles », et puissent ainsi faire front à l'idée d'évolution. Les crises sociales, la «mondialisation », l'affaiblissement supposé ou réel des États, la décomposition des sociétés fonctionnant implicitement de façon holiste, tous ces facteurs combinés entre eux sont un excellent terreau pour de tels mouvements religieux et idéologiques parce qu'ils recentrent les croyances. Le créationnisme fait partie d'un mouvement beaucoup plus large de retour critique sur les trois grandes figures du XIX ${ }^{\mathrm{e}}$ siècle, qui ont constitué au XX ${ }^{\mathrm{e}}$ les formes principales de la modernité : Darwin, Freud et Marx. Les deux derniers ont d'abord fait l'objet de critiques très vives : la psychanalyse ne peut être considérée de façon directe et simple comme une thérapie; le

Auteur correspondant : afschmid@free.fr

A.-F. Schmid, philosophe, membre du comité de rédaction de NSS, est à l'initiative de ce dossier. Elle enseigne la philosophie des sciences et l'épistémologie à l'INSA de Lyon et est membre $\mathrm{du}$ Laboratoire de philosophie et d'histoire des sciences - Archives Henri Poincaré, UMR 7117 du CNRS. marxisme a été comme «balayé » avec le communisme par la chute du mur de Berlin et l'effondrement du gouvernement de l'URSS. Ne reste donc que Darwin. C'est là un résumé sans doute un peu rapide. Il voudrait seulement suggérer que le créationnisme n'est pas une anecdote, mais un épisode d'un mouvement conservateur beaucoup plus large, qui dépasse sans doute les créationnistes eux-mêmes, lequel est lié à des phénomènes de transformations sociales, éthiques, et pas seulement religieuses. La compréhension d'un tel phénomène passe par des analyses à la fois anthropologiques, sociales, géographiques, politiques, économiques et religieuses.

Sensible au fait que ce phénomène touche les campus scientifiques depuis maintenant quatre ou cinq ans, et à la nécessité de clarifier les rapports entre théorie de l'évolution et mouvement créationniste, la rédaction de NSS a proposé d'ouvrir un dossier interdisciplinaire sur cette question, centré dans un premier temps sur ce que les biologistes écriraient de leur spécialité et de l'évolution. Nous pensons qu'à partir de ces textes, nous pourrons préciser le statut de l'idée d'évolution et montrer comment le concept d'évolution est compris dans un contexte scientifique.

Pour introduire ce débat, nous procéderons en deux temps : nous décrirons d'abord la manière dont raisonnent et agissent les créationnistes, puis nous proposerons des hypothèses pour interpréter les articles que des biologistes ont envoyés à la rédaction. Ces textes, retravaillés à partir des débats qui ont eu lieu lors d'un atelier de travail, le 19 juin 2007, seront publiés en plusieurs étapes dans la rubrique «Forums». Nous attendons, dans un premier temps, les textes de Jean-Claude Mounolou et Françoise Fridlansky, Jacques Daillie, John Buckeridge, Jacqueline Laurent, Christian Biémont, Pierre Clément et Bernard Hubert. 


\section{Le créationnisme contre Darwin : quels arguments ?}

Comment procèdent les créationnistes dans leur attaque contre Darwin? Leur premier geste est de mettre sur le même plan la Genèse et l'ouvrage de Darwin De l'origine des espèces par sélection naturelle ou Des lois de transformation des êtres organisés (1866). Deux méthodes concourent à cet effet. En premier lieu, ils tentent de donner une apparence scientifique à la Genèse, en rassemblant tous les faits, archéologiques, paléontologiques, qui peuvent, selon eux, accréditer certaines phrases de ce livre. Leur problème est de faire de la Genèse un texte descriptif. Les faits qu'ils évoquent n'ont aucun lien entre eux, si ce n'est justement d'être présentés comme faits. Mais ce n'est en aucune façon une démarche véritablement scientifique, les créationnistes mettant simplement en relation des fragments de texte avec quelques faits ou hypothèses archéologiques ou paléontologiques traitées comme faits. En second lieu, ils cherchent à montrer que le texte de Darwin n'est pas une théorie scientifique, mais une théorie philosophique. Les arguments utilisés pour le démontrer viennent directement de Karl Popper, l'un des rares épistémologues connus des scientifiques, et à qui ils font confiance. Popper avance comme critère de scientificité d'une proposition scientifique la possibilité de la réfuter, s'opposant ainsi aux vérificationnistes du cercle de Vienne. La proposition des philosophes des sciences de qualifier de scientifique toute proposition dès lors qu'elle est vérifiable lui paraissait trop simple, et surtout ne pas pouvoir traiter les problèmes épistémologiques posés par les théories de la relativité. Mais Popper a voulu faire d'une pierre deux coups, il a généralisé son critère pour montrer que les théories de Freud et de Marx n'étaient pas scientifiques, parce que non réfutables. Les créationnistes ont vu rapidement ce qu'ils pouvaient tirer de cet argument. Ils en concluent que la théorie de l'évolution n'est pas réfutable, la mettant par là même au niveau d'une théorie philosophique. Or, on notera avec amusement que les scientifiques les mieux armés admettent la démarche poppérienne, ce qui rend plus pervers encore les arguments créationnistes. Pour résumer ces méthodes, on peut dire que les créationnistes sont « vérificationnistes » quand ils parlent de la Genèse, mais qu'ils sont «réfutationnistes » quand ils s'attaquent à Darwin. À partir de cette double méthode, de « vérification » de la Genèse par référence à quelques faits admis et de réduction de la théorie de Darwin à une théorie philosophique, il leur est possible de dire qu'il y a deux façons d'expliquer l'apparition de la vie et ses transformations : celle qui relève de la Bible et celle qui relève de Darwin, et que chacun est libre de choisir. Cela donne aux créationnistes une apparence de libéralisme qui trompe les étudiants, parce qu'ils ne sont pas assez formés à l'épistémologie. Tout ce qu'ils écrivent par ailleurs sur les démarches scientifiques semble vraisemblable, lorsqu'ils sont dans la droite ligne des propositions de Popper. Ainsi la mécanique et la physique sont-elles acceptées par eux comme sciences à part entière, mais la biologie est considérée comme une demi-science reposant sur une théorie philosophique. De fait, la biologie est une science plus récente que la mécanique. Mais l'argument n'en révèle pas moins une profonde ignorance de l'histoire de cette dernière et de son inventivité pour mettre en place ses concepts fondamentaux. Les étudiants restent très mal armés devant de tels raisonnements.

Un autre argument des créationnistes est d'introduire des nuances dans leur refus de l'évolution. Il y a des domaines où elle est admise - par exemple, la microévolution plutôt que la macroévolution. Ils admettent qu'un individu puisse être modifié par son histoire et son environnement, mais ils excluent les modifications d'une espèce à une autre. Ou encore, ils admettent l'évolution dans le domaine de ce que l'on peut observer, mais la considèrent inacceptable en biologie moléculaire. Tout y est, selon eux, trop complexe pour que l'«émergence » de nouvelles propriétés puisse être l'effet du hasard. C'est l'idée de Behe, dont parle Ruse dans l'article qui suit ${ }^{1}$. La critique de Darwin est massive, pourtant elle se fait avec des nuances et des distinctions qui rendent plus délicate la critique du créationnisme. L'un des problèmes est que les échelles de temps sont finalement rapportées au présent - à ce que nous pouvons observer - et que l'on suppose que l'évolution a des effets sur des «individus " plutôt que sur des populations, ou sur de très larges échelles de temps. Le créationnisme est une position anthropocentrée - c'est l'homme qui est au centre et même individu-centrée - c'est chaque individu qui est concerné -, même si le concept de population peut y être connu. Les arguments contre l'évolution reposent sur l'idée que Dieu prend soin des personnes et des individus, et s'opposent à celle que la science doit les prendre pour objet. Le concept d'évolution ne porte pas sur les individus, ni sur leur filiation proche. Par contre, le mélange instable de religion et de morceaux d'évolution proposé par le créationnisme, sans distinction d'échelles, finit par identifier les hommes tels que nous les voyons et sentons comme les objets donnés de l'évolution.

Enfin, le créationnisme peut prendre une allure politique. Les laboratoires de biologie ainsi que de nombreux lycées ont été récemment inondés ${ }^{2}$ par l'envoi, depuis l'Allemagne, d'un livre de luxe intitulé Atlas de la création, grand et lourd comme un incunable, écrit par un auteur turc, Haroun Yahya. Cet ouvrage, qui est annoncé comme le premier d'une série de sept volumes, met en parallèle

\footnotetext{
1 Voir, dans ce numéro, le texte de Michael Ruse, «Intelligent Design Theory ». Par ailleurs, un compte rendu de son ouvrage The Evolution-Creation Struggle, par Jean-Claude Mounolou, figure dans la rubrique «Lectures - Ouvrages en débat ».

2 Par exemple, le laboratoire Biométrie et biologie évolutive de l'université de Lyon 1 en a reçu dix exemplaires!
} 
des photographies de fossiles et d'espèces actuelles pour montrer la stabilité des espèces. Vers la page 580 de ce tome qui en compte 800, l'auteur compare les darwinistes avec le communisme et l'hitlérisme qu'ils auraient inspirés, parce que la vie serait vue par Darwin comme un conflit, là où il parlait de "struggle for life », c'est-àdire de lutte pour la vie. Il y est même suggéré que les attentats du 11 septembre seraient le fait de darwinistes et non d'intégristes. Un livre d'un tel luxe montre à quel point les créationnistes disposent d'argent et de soutiens politiques et religieux.

L'ouvrage rend évidentes les raisons de l'accord entre les diverses religions sur un tel sujet : les arguments créationnistes venant des musulmans ou des protestants sont à peu près les mêmes. Sur nos campus, le créationnisme, qui était d'abord le fait des évangéliques, devient celui de musulmans. Il est probable qu'il pourra être un point d'accord de mouvements ou d'individus des religions monothéistes, même si le pape Jean-Paul II s'était rallié au darwinisme, ou du moins ne lui a pas fait obstacle.

\section{Les représentations de l'évolution chez les biologistes}

Longtemps, en France, on a cru que le concept ou la théorie de l'évolution était une sorte d'évidence, si bien qu'il était peu discuté, si ce n'est par quelques historiens ou philosophes des sciences. La tradition des Lumières a construit la confiance dans les sciences, de telle façon qu'elles pouvaient être admises sans toujours être élucidées. La lutte contre $1^{\prime}$ « obscurantisme» passe traditionnellement, en France, par une référence plus ou moins élaborée à la tradition des Lumières. Ce nouvel épisode montre que cette référence n'est pas du tout suffisante et que le contexte scientifique a changé.

Pour clarifier ce rapport création/évolution, nous avons demandé à des biologistes de s'exprimer sur la place de l'évolution dans leur discipline ou leur spécialité, et certains ont bien voulu écrire pour NSS. Les textes reçus soulignent tous l'importance de l'évolution, mais s'arrêtent souvent à ce constat. Redondants sur certains points et pas toujours clairs sur le concept d'évolution, ils ne pouvaient donc être publiés tels quels. Il fallait une journée de travail, où chacun des auteurs prenne la parole devant les autres. À partir de l'ensemble de ces articles, nous avons pu formuler des hypothèses sur la façon dont les biologistes comprennent l' «évolution » du point de vue de son statut épistémologique. Il semble qu'il y ait trois grandes postures, non exclusives, parmi les scientifiques - quoique ce choix ne se voie parfois que de façon implicite :

1. Certains comprennent l'évolution comme un fait scientifique. Elle n'est ni un concept ni une théorie, mais un fait scientifique fondamental. Les concepts seraient ceux de variation, de sélection naturelle. Darwin a mis en évidence un fait fondamental sans lequel la biologie est impossible. L'idée d'un «fait » de l'évolution signifie qu'il a été imposé aux scientifiques et qu'il n'a rien d'une fantaisie arbitraire, dont l'un des buts serait de s'opposer aux religions.

2. D'autres pensent que la théorie de l'évolution est une théorie scientifique au même titre que la mécanique de Newton. L'ignorance de l'histoire des sciences conduit à ce que l'on ne voit plus les difficultés qu'il $\mathrm{y}$ a eu à mettre en place cette dernière, à formuler en particulier de façon générale le principe d'inertie, à surmonter les arguments métaphysiques pour parler $\mathrm{d}^{\prime}$ " attraction ». L'évolution est une théorie et non pas un fait, capable d'unifier les concepts. C'est, par exemple, la posture de Jean-Marie Legay.

3. D'autres encore pensent que l'évolution est une métathéorie, c'est-à-dire une théorie qui permet de mettre en relation les diverses disciplines de la biologie. Elle permet d'unifier les rapports entre la génétique, la physiologie, l'embryologie, l'étude des systèmes biologiques, etc. Elle permet de donner sens à cette multiplicité de disciplines et d'en garantir l'unité et la cohérence. La théorie de l'évolution n'est pas au niveau des autres théories. C'est, par exemple, la position de Paul Nardon.

Les positions 1 et 3 peuvent être concomitantes. La position 1 peut aller avec l'idée qu'il y aura finalement une théorie unifiée de la biologie, comme il y a une théorie unifiée en physique. Cela suppose que la biologie se développera d'une façon plus «mathématique » et moins « expérimentale » qu'elle ne le fait maintenant.

D'un point de vue épistémologique, les trois positions peuvent être soutenues, mais peut-être pas exactement en même temps. Hanson a bien montré qu'une loi pouvait être traitée comme une définition, à condition qu'on ne lui demande pas de prédire. Elle sera utilisée comme telle dans un type de présentation, et autrement ailleurs. De l'évolution, on peut dire qu'il peut en être fait usage en fonction de ces trois positions épistémologiques, à condition que la démarche soit à chaque fois cohérente.

Il nous semble que les créationnistes s'attaquent à l'idée d'évolution comme théorie scientifique. Ils écrasent la notion d'évolution, au sens où ils n'en reconnaissent pas tous les usages scientifiques qui peuvent être faits et ne lui confèrent qu'un statut de théorie philosophique. C'est une situation qui demande éclaircissements et développements. Les articles de Michael Ruse et de Kathryn $\mathrm{Tabb}^{3}$, tous deux philosophes et historiens de la biologie, nous montrent comment sont mêlées les critiques du créationnisme et les conditions historiques et philosophiques dans lesquelles est apparue et dans lesquelles

\footnotetext{
3 Voir, dans ce numéro, les textes de Michael Ruse, loc. cit., et de Kathryn Tabb, «La téléologie de Darwin ».
} 
se développe actuellement la théorie de Darwin. C'est pourquoi il importe que les biologistes s'expriment de la façon la plus argumentée et la plus précise possible sur Darwin, sur l'évolution dans la science actuelle. Cela nous permettra de mettre à sa juste place le discours des créationnistes ou de l'ID.

\section{Le créationnisme profite d'une crise de la représentation des sciences}

La question du créationnisme, qu'il soit « dur » ou adouci sous la forme de ID, arrive donc sur nos campus, y compris parmi les étudiants de biologie. Mais comment interpréter cet engouement? La question n'est pas de savoir si l'on peut être scientifique et croyant à la fois, quelle que soit la religion ou la confession. Cela est manifestement possible, et bien des savants et des scientifiques ont mené ou mènent parfaitement leur carrière scientifique tout en étant engagés religieusement. Notre hypothèse est que le problème ne tient pas à la croyance religieuse $\mathrm{du}$ scientifique, mais aux modifications profondes qui ont affecté la représentation philosophique et sociale des sciences et l'ont conduite à une véritable crise. Il importe $\mathrm{d}^{\prime}$ analyser et de clarifier cette crise, afin qu'elle n'atteigne pas les sciences elles-mêmes. Pour cela, il ne suffit plus $\mathrm{d}$ 'invoquer les Lumières, il faut repenser le concept de science.

Cette crise de la représentation des sciences a plusieurs causes, qu'il faut connaître pour pouvoir évaluer l'impact du créationnisme.

1. Durant tout le $X X^{e}$ siècle, l'épistémologie a cherché à établir des critères à la science (vérificationnisme, réfutabilité, logique des programmes de recherche, etc.). À la fin du siècle, on s'aperçoit que les critères habituellement retenus ne sont pas suffisants ou qu'ils ne «fonctionnent » pas de façon universelle, ou encore que l'on n'a pas de critères. Quel que soit le choix de chacun, scientifique ou épistémologue, on ne pense plus qu'un critère simple puisse suffire à distinguer ce qui est science de ce qui ne l'est pas. Tout mélange de science avec un autre ordre du savoir peut paraître acceptable - et cela est une condition de légitimation des mouvements de pensée tel le créationnisme.

2. L'un des effets de cette situation est que l'on a cherché à aborder les sciences par leur fonctionnement social (Social Studies), en laissant tomber l'épistémologie ou en remplaçant une épistémologie des modèles par la «technologie». Comment se forment les « dogmes » scientifiques? Comment aboutit-on à des résultats scientifiques ? Comment pose-t-on des questions pertinentes? À travers une pratique sociale de laboratoire, répond-on, et ce que l'on concevait jusqu'alors comme résultat « objectif » devient le simple effet d'un accord au sein d'une communauté parlant un langage spécialisé, que ne comprennent pas ceux qui ne font pas partie de la communauté. La science est un «fait social », mais elle n'est pas seulement cela.

3. Cette réduction contemporaine a un point commun avec les conceptions classiques de la science. On conçoit celle-ci comme un donné, comme les géomètres deux siècles plus tôt pouvaient concevoir le point, la droite, le plan. Or, actuellement et plus qu'avant, la science nous est donnée mélangée à des techniques, des technologies, de l'éthique, de la philosophie. Certains expliquent ces mélanges par d'autres mélanges, dont le concept de technologie. Nous pensons qu'il convient plutôt de formuler des hypothèses sur ce que sont la science, la philosophie, la technique, etc. De telles hypothèses doivent être minimales, de façon à ne pas exclure des aspects de la science que les caractérisations épistémologiques plus compliquées risquent d'occulter. Par exemple, les caractérisations classiques de la science comme théorie, qui sont tout à fait pertinentes dans leur ordre, ne permettent pas de prendre en compte ce qui relève de la modélisation, de la conception, des simulations informatiques. Il faut donc élaborer des hypothèses plus " minimales », qui n'excluent ni les théories ni les modélisations.

4. Il y a eu, sur les modèles et la modélisation, une quantité de travaux épistémologiques assez récents, que les sociologues de la science ne prennent pas en compte, si ce n'est à travers la sociologie. Ils n'évoquent le plus souvent l'épistémologie qu'au travers de "l'épistémologie des théories ». Du coup, ils négligent les travaux d'épistémologie sur les autres aspects des sciences. Il est nécessaire de reprendre toute la question de la caractérisation du phénomène "science » et de chercher la juste place de la sociologie, sans qu'elle remplace ou occulte les travaux d'épistémologie.

5. Toutes ces réductions actuelles des sciences à l'épistémologie des théories, de l'épistémologie à la sociologie, tiennent à une autre confusion sur la question de l'objectivité scientifique. Le concept d'objectivité est un concept qui vient de la philosophie et qui, comme bien d'autres, a été projeté sur les sciences. Si l'on réduit cette objectivité à un accord des scientifiques, on ne réduit pas seulement la science à un fait social, on fait plus : on mélange science et philosophie. Les relations entre sciences et philosophies sont à penser autrement qu'on ne l'a fait jusqu'à maintenant, et les interprétations philosophiques des sciences doivent être analysées d'une façon nouvelle, en admettant une autonomie relative aussi bien des philosophies que des sciences. Il ne suffit pas de s'en remettre à la tradition des Lumières et de l'Encyclopédie. La critique de l'objectivité scientifique ne touche pas la science elle-même, mais bien les relations entre sciences et philosophies. 
Ce sont là quelques aspects de la crise actuelle du concept de science. On voit son incidence en philosophie et dans les représentations sociales des sciences (de la science?). Le créationnisme en profite. Puisque nous n'avons plus de critères de définition des sciences, il est plus facile d'argumenter pour montrer le caractère philosophique plutôt que scientifique de certains concepts fondamentaux de la biologie.

Pour cette première livraison, nous avons choisi des textes de philosophes et historiens de la biologie. Dans les numéros suivants, nous publierons les articles des biologistes, retravaillés à partir de leur discussion commune, le 19 juin 2007.

\section{Remerciements}

Je remercie le comité de rédaction de NSS de m'avoir fait des suggestions pour la rédaction de ce texte de cadrage, en particulier Claudine Friedberg, Nicole Mathieu et Marcel Jollivet, qui m'ont beaucoup aidée par leur lecture attentive et leurs remarques. 